\title{
Genetic Analysis of Mitochondrial Sorting from the MSC3 Mosaic Mutant of Cucumber
}

\author{
Lyle T. Wallace and Michael J. Havey \\ Agricultural Research Service, U.S. Department of Agriculture, Department of Horticulture, \\ University of Wisconsin, Madison, WI 53706
}

\begin{abstract}
AdDitIONAL INDEX wORDs. linkage mapping, mitochondrial genetics, quantitative trait locus
Abstract. Cucumber (Cucumis sativus) plants regenerated from cell cultures occasionally possess mosaic (MSC) phenotypes on cotyledons and leaves. Lines MSC3 and MSC16 have distinct MSC phenotypes and originated from plants regenerated from different cell-culture experiments established using a highly inbred wild-type cucumber. Both the mitochondrial (mt) DNA and MSC phenotype of cucumber show paternal transmission, and MSC3 and MSC16 have different mt coding regions at significantly lower copy numbers relative to wild-type plants. A nuclear locus, Paternal sorting of mitochondria (Psm), conditions a high proportion of wild-type progenies, specifically when MSC16 is crossed as the male with wild-type female plants. During this research, we identified plants that produced a high proportion of wild-type progenies in crosses with MSC3 as the male parent. Plants from an $F_{2}$ family were crossed with MSC3 as the male, progenies were scored for numbers of MSC vs. wild-type plants, and single-nucleotide polymorphisms (SNP) were identified for genetic mapping. A major quantitative trait locus on chromosome 3 was associated with a higher frequency of wild-type progenies from MSC3 as the male parent, and the 1.5-logarithm-of-odds interval for the most significant SNP was located $627 \mathrm{~kb}$ from Psm. These results reveal that separate genetic factors control sorting to the wild-type phenotype in progenies from crosses with different MSC parents. The identification of causal genes controlling mitochondrial sorting in cucumber should provide insight regarding nuclear-mitochondrial interactions affecting the prevalence of specific mitochondrial DNA in plants.
\end{abstract}

The mitochondrial (mt) DNA of cucumber (Cucumis sativus) has distinctive characteristics relative to most plants and include paternal transmission, a large size $(1.685 \mathrm{Mb})$, and a high degree of structural polymorphisms within cultivated germplasm (Alverson et al., 2011; Havey, 1997; Havey et al., 1998; Lilly and Havey, 2001). Because the three genomes in cucumber show differential transmission (maternal for chloroplast, paternal for mitochondrial, and biparental for nuclear), phenotypes and polymorphisms can be assigned to genomes by reciprocal crossing (Havey et al., 1998). Passage of the highly inbred line B of cucumber through cell culture occasionally produces regenerated plants with a mosaic (MSC) phenotype on cotyledons and leaves (Malepszy et al., 1996). Genetic studies revealed that the MSC phenotype is paternally transmitted (Malepszy et al., 1996), and that MSC lines from independent cell-culture experiments have different rearrangements and under-represented regions in their mt DNA compared with their wild-type progenitor B (Bartoszewski et al., 2004, 2007; Ładyżyński et al., 2002). Mosaic cucumbers MSC3 and MSC16 were regenerated from different cell cultures and have different regions of their mt DNA at significantly lower copy numbers relative to inbred B (Del Valle-

Received for publication 13 Apr. 2021. Accepted for publication 19 May 2021. Published online 6 July 2021.

We gratefully acknowledge the University of Wisconsin Biotechnology Center DNA Sequencing Facility for providing sequencing facilities and bioinformatic services.

This research was supported by the U.S. Department of Agriculture, National Institute of Food and Agriculture (grant 2016-67013-24590).

Names are necessary to report factually of available data; however, the U.S. Department of Agriculture (USDA) neither guarantees nor warrants the standard of the product, and the use of the name by the USDA implies no approval of the product to the exclusion of others that may also be suitable. M.J.H. is the corresponding author. E-mail: mjhavey@wisc.edu.

This is an open access article distributed under the CC BY-NC-ND license (https://creativecommons.org/licenses/by-nc-nd/4.0/).
Echevarria et al., 2015). MSC3 has fewer copies of the polycistronic region encoding NADH dehydrogenase subunit 5 and ATP synthase subunit 4, whereas MSC16 has fewer copies of ribosomal protein S7 (Del Valle-Echevarria et al., 2015).

When wild-type cucumber plants are crossed as the female with MSC3 or MSC16 as the male, essentially all progenies show the MSC phenotype (Lilly et al., 2001; Malepszy et al., 1996). The reciprocal cross (MSC3 or MSC16 as the female parent with wild-type plants as the male) produces only wildtype progenies. Cucumber germplasm was screened by crossing wild-type plants with MSC16 as the male, and individual plants from the U.S. Department of Agriculture (USDA) Plant Introduction (PI) 401734 produced significantly higher numbers of wild-type progenies (Havey et al., 2004). A nuclear locus, Paternal sorting of mitochondria (Psm), controls sorting to the wild-type phenotype in progenies from crosses with MSC16 as the male parent (Havey et al., 2004; Lilly et al., 2001). When the female parent is homozygous for the Psm+ allele, progeny from the crossing of MSC16 as the male show the MSC phenotype. When the female parent is heterozygous at Psm (+/-), crossing with MSC16 as the male will produce approximately equal numbers of wild-type and MSC progenies. When female plants homozygous for the rarer Psm- allele are crossed with MSC16 as the male, essentially all progeny are wild-type (Havey et al., 2004). The Psm locus maps to chromosome 3 of cucumber (Al-Faifi et al., 2008; Calderon et al., 2012), and pentatricopeptide repeat (PPR) 336 has been proposed as a candidate gene for Psm (Del Valle-Echevarria et al., 2016). PPR proteins are targeted to the organelles, where they are involved with post-transcriptional modifications and translation (Barkan and Small, 2014). PPR336 has been shown to stabilize mitochondrial polyribosomes in Arabidopsis thaliana (Uyttewaal et al., 2008) and may help to maintain ribosomal function despite fewer copies of the ribosomal protein S7 gene and its 
transcript in MSC16 mitochondria (Del Valle-Echevarria et al., 2016).

When plants with different genotypes at Psm were crossed with both MSC3 and MSC16 as males, segregation of MSC vs. wild-type progenies differed between families from these two MSC parents (Del Valle-Echevarria et al., 2016). Because MSC3 and MSC16 have different under-represented regions of their mt DNA, we were interested in determining whether the Psm locus has a role in sorting to wild type from different MSC mitochondrial mutants. The goals of this research were to study the genetic basis of mitochondrial sorting in progenies from crosses with MSC3 and to determine if independent genetic control of mitochondrial sorting from different MSC parents exists.

\section{Materials and Methods}

Plants were grown in greenhouses at the University of Wisconsin in 9-L pots containing a soilless mix (Pro-Mix HP Mycorrhizae; Premier Tech Horticulture, Quakertown, PA) at temperatures between 26 and $31^{\circ} \mathrm{C}$ and fertilized twice per week with 20N-8.7P-16.6K (Peters Professional 20-10-20; Everris, Dublin, OH). PI 401734 from the USDA germplasm collection was previously identified as producing higher frequencies of wild-type progenies in crosses with MSC16 as the male parent (Havey et al., 2004). Cucumis sativus var. hardwickii is a feral relative of cucumber and produces only mosaic progenies from crosses with MSC16 as the male (Calderon et al., 2012). A single plant from PI 401734 was crossed as the female with $C$. sativus var. hardwickii, one hybrid was self-pollinated to generate a segregating family, and $\mathrm{F}_{2}$ plants were crossed with MSC16 as the male. Frequencies of wild-type vs. mosaic progenies were used to map the Psm locus (Calderon et al., 2012). Plants from this same family that were homozygous at Psm $(+/+$ or $-/-)$ were crossed with both MSC3 and MSC16 as the males. Seeds from these crosses were grown in sterilized vermiculite at $30^{\circ} \mathrm{C}$ and progenies were scored as wild-type vs. mosaic. One plant (C10630) with the genotype Psm-/- showed higher numbers of wild-type progenies in crosses with MSC3 as the male, and it was crossed as the male to a plant from the doubled haploid (DH) 9930 (Shen et al., 2015). One hybrid was self-pollinated to produce $\mathrm{F}_{2}$ progenies that were crossed as the female with MSC3 as the male. Fifty seeds from each family were grown and cotyledons were scored as wild type vs. mosaic. Two fruits were produced from many of the $\mathrm{F}_{2}$ plants, and the numbers of wildtype and mosaic progenies from each fruit were averaged. The proportion of wild-type progenies in each family from crossing with MSC3 was calculated after requiring at least 50\% germination of seed and used for genetic analyses.

DNA was isolated from $80 \mathrm{mg}$ of lyophilized leaves from parental plants and $\mathrm{F}_{2}$ progenies using a kit (NucleoSpin Plant II Midi kit; Macherey-Nagel, Düren, Germany). Concentrations were determined spectrophotometrically (NanoDrop 1000; Thermo Fisher Scientific, Waltham, MA). DNA of the two parents and $122 \mathrm{~F}_{2}$ progenies were digested with ApeKI (Wang et al., 2018) and genotyped by sequencing (Davey et al., 2011) at the University of Wisconsin Biotechnology Center (NovaSEq. 6000; Illumina, San Diego, CA). Skewer software (Jiang et al., 2014) was used to trim the $3^{\prime}$ end of fragments until a Phred quality of 20 was reached. The SNPs were identified using TASSEL 5.0 GBS Discovery Pipeline (Glaubitz et al., 2014) and the cucumber 9930 version 3.0 reference genome (Li et al., 2019) as described by Wang et al. (2018). SNPs were filtered based on $\leq 50 \%$ missing data and minor allele frequencies $\geq 5 \%$. Linkages among SNPs were detected using JoinMap version 5.0 (Van Ooijen, 2018) with the maximum likelihood mapping algorithm and an independence logarithm of odds (LOD) greater than 6.0 for linkage. Quantitative analysis of mitochondrial sorting was completed using the R/qtl package (Broman and Sen, 2009; Broman et al., 2003) in R Studio (R Foundation for Statistical Computing, Vienna, Austria). Quantitative trait loci (QTL) were detected using the scanone, scantwo, and stepwiseqtl functions, and a LOD threshold at $P=0.05$ was computed after 1000 permutations. The fitqtl and refineqtl functions were used to estimate effects of the candidate QTL, percentage of the phenotypic variation explained by the QTL, and the LOD-1.5 interval surrounding the most significant SNP.

\section{Results and Discussion}

Different frequencies of wild-type vs. MSC progenies were observed from crossing the same female plants with MSC3 and MSC16 as males. For 11 plants with the $P s m+/+$ genotype, crosses with MSC16 as the male produced very few (average proportion, 0.01) wild-type progenies (Table 1). When the same plant was crossed with MSC3, we observed varying numbers of wild-type and mosaic progenies (Fig. 1), with an average proportion of $0.27 \pm 0.34$ wild-type progenies (Table 1). We crossed 33 plants that were Psm-/- with both MSC3 and MSC16 as males and observed that all families from crosses with MSC16 showed high proportions of wild-type progenies (average, $0.96 \pm 0.04$ ) (Table 1). Crosses of the same plants with MSC3 produced families with variable numbers of wildtype and mosaic progenies, with an average proportion of wild-type of $0.58 \pm 0.26$ (Table 1). Different segregations for wild-type vs. MSC progenies from crosses with MSC3 and MSC16 indicate that the Psm locus may not control sorting to the wild-type phenotype in progenies from crosses with different MSC parents.

All of the 95 progenies from the cross of DH9930 with MSC3 as the male had the mosaic phenotype (Fig. 1). Progenies from crossing plant $\mathrm{C} 10630$ with MSC3 as the male produced an average of $0.88 \pm 0.03$ wild-type progenies. DH9930 was crossed with $\mathrm{C} 10630$, and a single $\mathrm{F}_{1}$ plant was self-pollinated to produce an $\mathrm{F}_{2}$ family. A total of $122 \mathrm{~F}_{2}$ progenies were crossed with MSC3 as the male, and the proportions of wildtype progenies (Fig. 1) ranged from 0.00 to 0.95 (Supplemental Table 1), with an average of $0.44 \pm 0.25$.

A total of 405,339,314 demultiplexed sequencing reads were produced across all DNAs, with an average number of 3,166,713 reads per DNAs sample. Results of three $\mathrm{F}_{2}$ DNA were discarded because of low numbers $\left(<1 \times 10^{5}\right)$ of demultiplexed reads. Reads were aligned to the 9930 version 3.0 reference sequence and 15,497 SNPs were identified, with an average number of 2214 SNPs per chromosome. Genetic mapping was completed using 4633 SNPs that fit the expected 1:2:1 ratio $(P>0.05)$ and after randomly selecting one SNP from groups of SNPs showing $>95 \%$ identical genotypes across the progeny DNAs. Genetic mapping at LOD 6.0 produced seven linkage groups corresponding to the seven chromosomes of cucumber. Overall, there was close agreement between genetic linkages among SNPs and their positions in the reference sequence; however, there were 
Table 1. Progenies with the wild-type (WT) vs. mosaic (MSC) phenotype and proportion of WT (Prop WT) progenies resulting from crosses of female cucumber plants with homozygous genotypes $(+/+$ or $-/-)$ at the Psm locus with both MSC3 and MSC16 as male parents.

\begin{tabular}{|c|c|c|c|c|c|c|}
\hline \multirow[b]{3}{*}{$\begin{array}{l}\text { Genotype of } \\
\text { female parent }\end{array}$} & \multicolumn{6}{|c|}{ Male parent } \\
\hline & \multicolumn{3}{|c|}{ MSC3 } & \multicolumn{3}{|c|}{ MSC16 } \\
\hline & \multicolumn{3}{|c|}{ Progenies (no.) } & \multicolumn{3}{|c|}{ Progenies (no.) } \\
\hline$\overline{P s m+/+}$ & 0 & 48 & 0.00 & 0 & 48 & 0.00 \\
\hline $\mathrm{Psm}+/+$ & 0 & 52 & 0.00 & 2 & 48 & 0.04 \\
\hline$P s m+/+$ & 1 & 49 & 0.02 & 0 & 54 & 0.00 \\
\hline$P s m+1+$ & 1 & 51 & 0.02 & 2 & 46 & 0.04 \\
\hline$P s m+1+$ & 2 & 48 & 0.04 & 1 & 49 & 0.02 \\
\hline $\mathrm{Psm}+/+$ & 3 & 46 & 0.06 & 0 & 54 & 0.00 \\
\hline$P s m+/+$ & 5 & 48 & 0.09 & 0 & 48 & 0.00 \\
\hline$P s m+/+$ & 29 & 21 & 0.58 & 0 & 51 & 0.00 \\
\hline$P s m+/+$ & 33 & 17 & 0.66 & 0 & 46 & 0.00 \\
\hline$P s m+/+$ & 35 & 13 & 0.73 & 0 & 51 & 0.00 \\
\hline$P s m+/+$ & 45 & 10 & 0.82 & 0 & 46 & 0.00 \\
\hline Total & 154 & 403 & 0.28 & 5 & 541 & 0.01 \\
\hline Psm-l- & 0 & 50 & 0.00 & 45 & 4 & 0.92 \\
\hline Psm-l- & 0 & 46 & 0.00 & 47 & 3 & 0.94 \\
\hline Psm-/- & 8 & 41 & 0.16 & 48 & 2 & 0.96 \\
\hline Psm-/- & 10 & 39 & 0.20 & 50 & 0 & 1.00 \\
\hline Psm-/- & 13 & 36 & 0.27 & 46 & 2 & 0.96 \\
\hline Psm-/- & 22 & 26 & 0.46 & 46 & 3 & 0.94 \\
\hline Psm-/- & 23 & 27 & 0.46 & 39 & 9 & 0.81 \\
\hline Psm-l- & 23 & 27 & 0.46 & 39 & 3 & 0.93 \\
\hline Psm-/- & 24 & 26 & 0.48 & 53 & 2 & 0.96 \\
\hline Psm-/- & 25 & 26 & 0.49 & 49 & 1 & 0.98 \\
\hline Psm-/- & 25 & 25 & 0.50 & 25 & 3 & 0.89 \\
\hline Psm-/- & 26 & 27 & 0.49 & 41 & 5 & 0.89 \\
\hline Psm-/- & 26 & 24 & 0.52 & 47 & 4 & 0.92 \\
\hline Psm-/- & 26 & 23 & 0.53 & 47 & 2 & 0.96 \\
\hline Psm-/- & 26 & 22 & 0.54 & 46 & 2 & 0.96 \\
\hline Psm-l- & 27 & 23 & 0.54 & 47 & 0 & 1.00 \\
\hline Psm-l- & 28 & 20 & 0.58 & 48 & 2 & 0.96 \\
\hline Psm-/- & 29 & 21 & 0.58 & 47 & 3 & 0.94 \\
\hline Psm-/- & 31 & 18 & 0.63 & 47 & 2 & 0.96 \\
\hline Psm-/- & 31 & 14 & 0.69 & 46 & 1 & 0.98 \\
\hline Psm-/- & 34 & 16 & 0.68 & 50 & 0 & 1.00 \\
\hline Psm-/- & 35 & 14 & 0.71 & 49 & 1 & 0.98 \\
\hline Psm-/- & 35 & 15 & 0.70 & 47 & 2 & 0.96 \\
\hline Psm-l- & 36 & 9 & 0.80 & 47 & 3 & 0.94 \\
\hline Psm-l- & 37 & 13 & 0.74 & 45 & 5 & 0.90 \\
\hline Psm-/- & 39 & 11 & 0.78 & 49 & 0 & 1.00 \\
\hline Psm-/- & 39 & 10 & 0.80 & 50 & 0 & 1.00 \\
\hline Psm-/- & 40 & 10 & 0.80 & 50 & 0 & 1.00 \\
\hline Psm-/- & 43 & 7 & 0.86 & 50 & 0 & 1.00 \\
\hline Psm-/- & 45 & 2 & 0.96 & 49 & 1 & 0.98 \\
\hline Psm-/- & 46 & 4 & 0.92 & 50 & 0 & 1.00 \\
\hline Psm-/- & 46 & 0 & 1.00 & 27 & 0 & 1.00 \\
\hline Psm-/- & 47 & 3 & 0.94 & 50 & 0 & 1.00 \\
\hline Total & 945 & 675 & 0.58 & 1516 & 65 & 0.96 \\
\hline
\end{tabular}

Psm $=$ Paternal sorting of mitochondria.

occasional positions at which the genetic linkages and genomic position did not agree, possibly because of mis-scoring of SNP genotypes across short genomic regions.

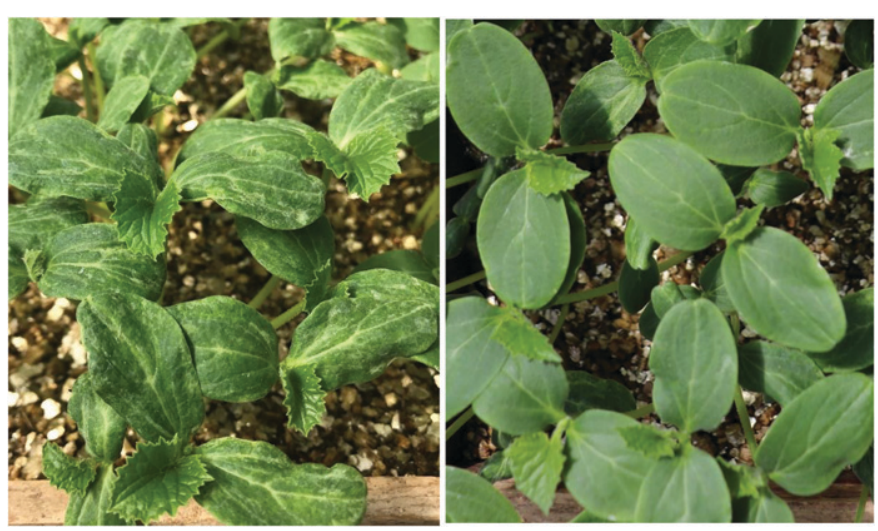

Fig. 1. Mosaic (MSC) progenies (left) from the crossing of cucumber doubled haploid 9930 as the female with MSC3 as the male. Wild-type progenies (right) were from the cross of an $\mathrm{F}_{2}$ plant with MSC3 as the male.

Quantitative analysis revealed one region on chromosome 3 associated with the proportion of wild-type progenies from crosses with MSC3 as the male (Fig. 2). The most significant SNP was at basepair (bp) 34,246,720 on chromosome 3, with a LOD score of 40.0 (LOD threshold $=4.87$ ); this explained $70.6 \%$ of the phenotypic variation. Additive and dominance effects were both significant $(P<0.05)$ and increased the numbers of wild-type progenies by $32.2 \%$ and $14.5 \%$, respectively. These attributes are consistent with a locus at which partially dominant alleles control sorting to the wild-type phenotype in progenies from MSC3 as the male parent and differ from the codominance of alleles at the Psm locus (Calderon et al., 2012; Havey et al., 2004).

The Psm locus controls sorting to wild type in progenies from crosses with MSC16 as the male (Calderon et al., 2012; Havey et al., 2004), and PPR336 has been proposed as the candidate gene for Psm (Del Valle-Echevarria et al., 2015). This gene is located from bp 33,618,502 to 33,619,719 on chromosome 3 in the 9930 reference sequence (Li et al., 2019). The LOD-1.5 interval surrounding the most significant SNP associated with sorting to wild-type progenies from crosses with MSC3 encompassed 134 kilobases $(\mathrm{kb})$ from bp 34,246,720 to 34,380,732 on chromosome 3; this region is located $627 \mathrm{~kb}$ from PPR336. There

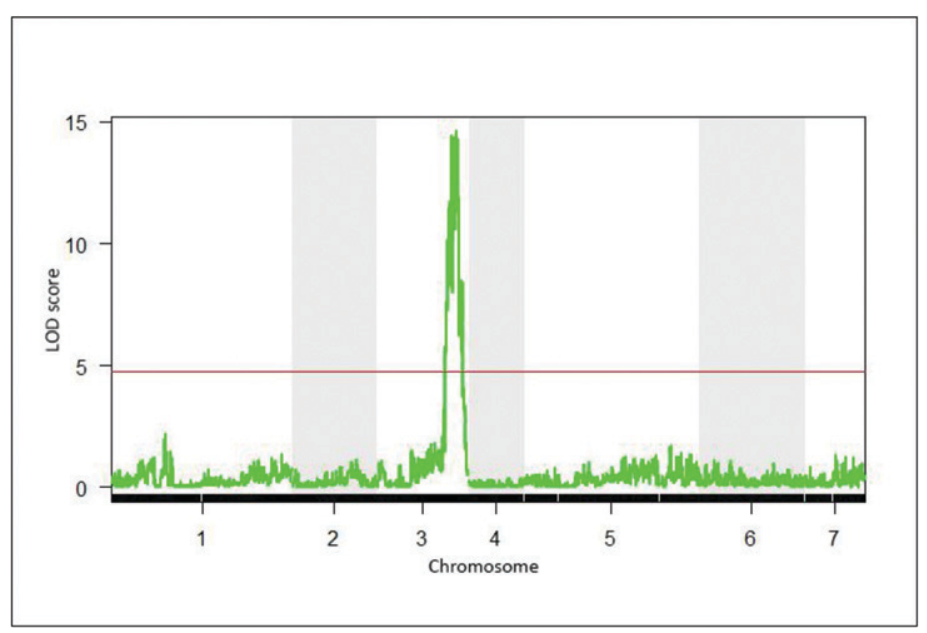

Fig. 2. Plot of logarithm of odds (LOD) scores (y-axis) for the sorting of wild-type progenies vs. marker positions across the seven chromosomes ( $\mathrm{x}$ axis) of cucumber. The genome-wide threshold as determined by permutation analysis at $P=0.05$ is shown by the red line at LOD 4.87 . 
Table 2. Locations and annotations of cucumber genes from version 3 of the Chinese Long 9930 genomic sequence (Li et al., 2019) across the logarithm-of-odds (LOD)-1.5 interval on chromosome 3 associated with proportions of wild-type and mosaic (MSC) progenies from crosses of $\mathrm{F}_{2}$ progenies as the female with $\mathrm{MSC} 3$ as the male and the corresponding most similar annotated genes in Arabidopsis thaliana.

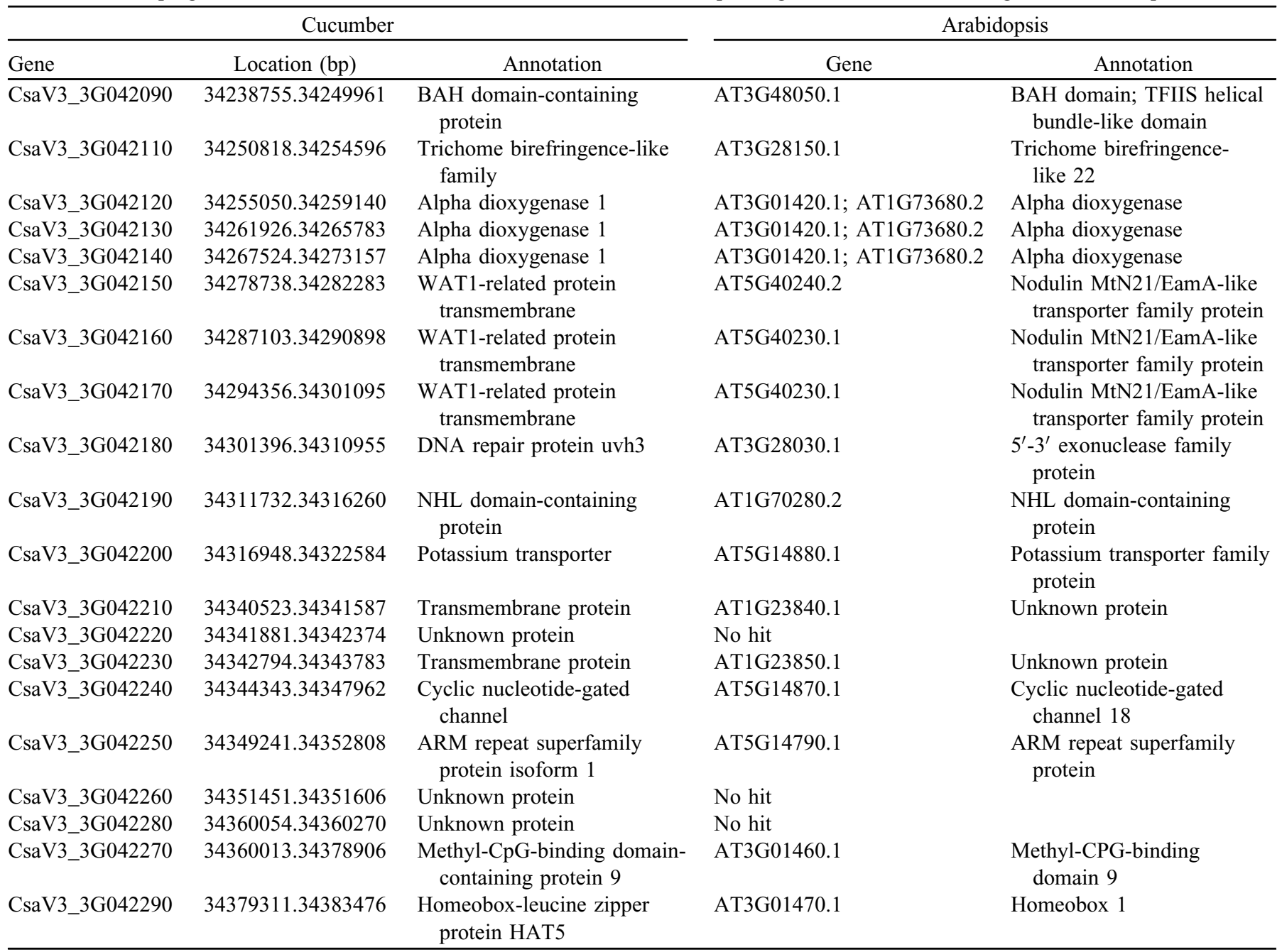

are 20 annotated genes (Table 2) in the 9930 reference sequence (Li et al., 2019) across the LOD-1.5 interval, of which two were identified as possible candidate genes. The first candidate is alpha-dioxygenase, which has three copies (CsaV3_3G042120, CsaV3_3G042130, and CsaV3_3G042140) in the genomic region of the QTL. Alpha-dioxygenase genes are important in lipid biochemical pathways in organelles because they add oxygen to lipids to produce oxylipins, which have roles in defense responses, senescence, and stress tolerance (Machado et al., 2015). The second candidate gene (CsaV3_3G042250) encodes for an isomer of the ARM repeat superfamily proteins, which have repetitive motifs similar to PPRs and are involved with plant cell physiology, stress, and development (Manisha and Pandey, 2016). Candidate genes involved with stress response may help to mitigate the deleterious effects of the MSC phenotype, thereby enhancing the survival of MSC progenies. Genetic variants that are less effective at mitigating the stress caused by MSC may allow relatively rare wild-type mitochondrial DNA to become more predominant, resulting in wild-type progenies.

This research demonstrates that different nuclear genomic regions affect sorting to the wild-type phenotype in progenies from different MSC male parents. Larger family sizes will be necessary to confidently identify the causal genes for mitochondrial sorting from MSC3. The eventual identification of genes controlling mitochondrial sorting in cucumber should provide insight regarding specific nuclear-mitochondrial interactions affecting the predominance of mitochondria delivered to progenies and subsequent effects on the growth and vigor of plants.

\section{Literature Cited}

Al-Faifi, S., J.D.F. Meyer, J. Garcia-Mas, A.J. Montforte, and M.J. Havey. 2008. Exploiting synteny in Cucumis for mapping of Psm: A unique locus controlling paternal mitochondrial sorting. Theor. Appl. Genet. 117:523-529, doi: 10.1007/s00122-008-0796-1.

Alverson, A.J., D.W. Rice, S. Dickinson, K. Barry, and J.D. Palmer. 2011. Origins and recombination of the bacterial-sized multichromosomal mitochondrial genome of cucumber. Plant Cell 23:2499-2513, doi: $10.1105 /$ tpc.111.087189.

Barkan, A. and I. Small. 2014. Pentatricopeptide repeat proteins in plants. Annu. Rev. Plant Biol. 65:415-442, doi: 10.1146/annurev-arplant050213-040159.

Bartoszewski, G., M.J. Havey, A. Ziółkowska, and S. Malepszy. 2007. The selection of mosaic (MSC) phenotype of cucumber (Cucumis sativus L.) through cell culture - A method for production of 
plant mitochondrial mutants. J. Appl. Genet. 48:1-9, doi: 10.1007/ BF03194652.

Bartoszewski, G., S. Malepszy, and M.J. Havey. 2004. Mosaic (MSC) cucumbers regenerated from independent cell cultures possess different mitochondrial rearrangements. Curr. Genet. 45:45-53, doi: 10.1007/s00294-003-0456-6.

Broman, K.W. and S. Sen. 2009. A guide to QTL mapping with R/qtl. Springer-Verlag, New York, NY.

Broman, K.W., H. Wu, S. Sen, and G.A. Churchill. 2003. R/qtl: QTL mapping in experimental crosses. Bioinformatics 19:889-890, doi: 10.1093/bioinformatics/btg112.

Calderon, C.I., B.S. Yandell, and M.J. Havey. 2012. Genetic mapping of paternal sorting of mitochondria in cucumber. Theor. Appl. Genet. 125:11-18, doi: 10.1007/s00122-012-1812-z.

Davey, J.W., P.A. Hohenlohe, P.D. Etter, J.Q. Boone, J.M. Catchen, and M.L. Blaxter. 2011. Genome-wide genetic marker discovery and genotyping using next-generation sequencing. Nat. Rev. Genet. 12:499-510, doi: $10.1038 / \operatorname{nrg} 3012$.

Del Valle-Echevarria, A.R., A. Kiełkowska, G. Bartoszewski, and M.J. Havey. 2015. The mosaic mutants of cucumber: A method to produce knock-downs of mitochondrial transcripts. Genes Genomes Genet. 5:1211-1221, doi: 10.1534/g3.115.017053.

Del Valle-Echevarria, A.R., W. Sanseverino, J. Garcia-Mas, and M.J. Havey. 2016. Pentatricopeptide repeat 336 as the candidate gene for paternal sorting of mitochondria (Psm) in cucumber. Theor. Appl. Genet. 129:1951-1959, doi: 10.1007/s00122-016-2751-x.

Glaubitz, C., T. Casstevens, F. Liu, R. Elshire, Q. Sun, and E. Buckler. 2014. TASSEL-GBS: A high capacity genotyping by sequencing analysis pipeline. PLoS One 9(2):e90346. < https://journals.plos.org/ plosone/article?id=10.1371/journal.pone.0090346 $>$.

Havey, M.J. 1997. Predominant paternal transmission of the mitochondrial genome in cucumber. J. Hered. 88:232-235, doi: 10.1093/ oxfordjournals.jhered.a023094.

Havey, M.J., J. McCreight, B. Rhodes, and G. Taurick. 1998. Differential transmission of the cucurbit organellar genomes. Theor. Appl. Genet. 97:122-128, doi: 10.1007/s001220050875.

Havey, M.J., Y.H. Park, and G. Bartoszewski. 2004. The Psm locus controls paternal sorting of the cucumber mitochondrial genome. J. Hered. 95:492-497, doi: 10.1093/jhered/esh081.

Jiang, H., R. Lei, D.W. Ding, and S. Zhu. 2014. Skewer: A fast and accurate adapter trimmer for next-generation sequencing paired-end reads. BMC Bioinformatics 15:1-12, doi: 10.1186/1471-2105-15-182.
Ładyżyński, M., W. Burza, and S. Malepszy. 2002. Relationship between somaclonal variation and type of culture in cucumber. Euphytica 125:349-356, doi: 10.1023/A:1016017825907.

Li, Q., H. Li, W. Huang, Y. Xu, Q. Zhou, S. Wang, J. Ruan, S. Huang, and Z. Zhang. 2019. A chromosome-scale genome assembly of cucumber (Cucumis sativus L.). Gigascience 8:giz072. <http:// cucurbitgenomics.org/organism/20>.

Lilly, J.W. and M.J. Havey. 2001. Short repetitive motifs contributed significantly to the huge mitochondrial genome of cucumber. Genetics 159:317-328.

Lilly, J.W., G. Bartoszewski, S. Malepszy, and M.J. Havey. 2001. A major deletion in the mitochondrial genome is transmitted with MSC phenotype of cucumber. Curr. Genet. 40:144-151, doi: 10.1007/ s002940100238.

Machado, L., A. Castro, M. Hamberg, G. Bannenberg, C. Gaggero, C. Castresana, and I. Ponce de León. 2015. The Physcomitrella patens unique alpha-dioxygenase participates in both developmental processes and defense responses. BMC Plant Biol. 15:45, doi: 10.1186/ s12870-015-0439-z.

Malepszy, S., W. Burza, and M. Śmiech. 1996. Characterization of a cucumber (Cucumis sativus L.) somaclonal variant with paternal inheritance. J. Appl. Genet. 37:65-78.

Manisha, S. and G.K. Pandey. 2016. Expansion and function of repeat domain proteins during stress and development in plants. Front. Plant Sci. 6:1218, doi: 10.3389/fpls.2015.01218.

Shen, J., R. Dirks, and M.J. Havey. 2015. Diallel crossing among doubled haploids of cucumber reveals significant reciprocal-cross differences. J. Amer. Soc. Hort. Sci. 140:178-182, doi: 10.1007/ s00122-018-03274-0.

Uyttewaal, M., H. Mireau, M. Rurek, K. Hammani, N. Arnal, M. Quadrado, and P. Giegé. 2008. PPR336 is associated with polysomes in plant mitochondria. J. Mol. Biol. 375:626-636, doi: 10.1016/j. jmb.2007.11.011.

Van Ooijen, J.W. 2018. JoinMap 5, software for the calculation of genetic linkage maps in experimental populations. Kyazma, Wageningen, the Netherlands.

Wang, X., K. Bao, U.K. Reddy, Y. Bai, S.A. Hammar, C. Jiao, T.C. Wehner, A.O. Ramírez-Madera, Y. Weng, R. Grumet, and Z. Fei. 2018. The USDA cucumber (Cucumis sativus L.) collection: Genetic diversity, population structure, genome-wide association studies, and core collection development. Hort. Res. 5:64, doi: 10.1038/ s41438-018-0080-8. 
Supplemental Table 1. Total numbers of cucumber progenies and proportions of wild-type (WT) vs. mosaic (MSC) progenies from crosses of $\mathrm{F}_{2}$ plants as the female with MSC3 as the male.

\begin{tabular}{|c|c|c|c|}
\hline \multirow[b]{2}{*}{$\mathrm{F}_{2}$ plant } & \multirow[b]{2}{*}{ Total progenies (no.) } & \multicolumn{2}{|c|}{ Proportion of progenies } \\
\hline & & WT & MSC \\
\hline C10627A & 99 & 0.44 & 0.56 \\
\hline C10627B & 93 & 0.37 & 0.63 \\
\hline C10627C & 50 & 0.00 & 1.00 \\
\hline C10627D & 98 & 0.39 & 0.61 \\
\hline $\mathrm{C} 10627 \mathrm{E}$ & 45 & 0.42 & 0.58 \\
\hline $\mathrm{C} 10627 \mathrm{~F}$ & 61 & 0.12 & 0.89 \\
\hline $\mathrm{C} 10627 \mathrm{G}$ & 99 & 0.01 & 0.99 \\
\hline $\mathrm{C} 10627 \mathrm{H}$ & 49 & 0.51 & 0.49 \\
\hline C10627I & 73 & 0.53 & 0.47 \\
\hline C10627J & 50 & 0.18 & 0.82 \\
\hline $\mathrm{C} 10627 \mathrm{~K}$ & 99 & 0.54 & 0.47 \\
\hline C10627L & 62 & 0.00 & 1.00 \\
\hline $\mathrm{C} 10627 \mathrm{M}$ & 92 & 0.49 & 0.51 \\
\hline $\mathrm{C} 10627 \mathrm{~N}$ & 31 & 0.71 & 0.29 \\
\hline $\mathrm{C} 10627 \mathrm{O}$ & 96 & 0.02 & 0.98 \\
\hline C10627P & 100 & 0.44 & 0.56 \\
\hline C10627Q & 98 & 0.56 & 0.44 \\
\hline C10627R & 70 & 0.81 & 0.19 \\
\hline C10627S & 49 & 0.45 & 0.55 \\
\hline $\mathrm{C} 10627 \mathrm{~T}$ & 18 & 0.28 & 0.72 \\
\hline $\mathrm{C} 10627 \mathrm{U}$ & 99 & 0.55 & 0.46 \\
\hline C10627V & 99 & 0.52 & 0.49 \\
\hline $\mathrm{C} 10627 \mathrm{~W}$ & 51 & 0.00 & 1.00 \\
\hline C10627X & 77 & 0.49 & 0.51 \\
\hline C10627Y & 80 & 0.50 & 0.50 \\
\hline C10627Z & 80 & 0.49 & 0.51 \\
\hline C10627AA & 74 & 0.60 & 0.41 \\
\hline $\mathrm{C} 10627 \mathrm{AB}$ & 75 & 0.57 & 0.43 \\
\hline $\mathrm{C} 10627 \mathrm{AC}$ & 84 & 0.67 & 0.33 \\
\hline C10627AD & 62 & 0.47 & 0.53 \\
\hline C10627AE & 54 & 0.48 & 0.52 \\
\hline C10627AF & 82 & 0.01 & 0.99 \\
\hline C10627AG & 81 & 0.49 & 0.51 \\
\hline C10627AH & 28 & 0.57 & 0.43 \\
\hline C10627AI & 85 & 0.44 & 0.57 \\
\hline C10627AJ & 56 & 0.32 & 0.68 \\
\hline C10627AK & 50 & 0.68 & 0.32 \\
\hline C10627AL & 62 & 0.26 & 0.74 \\
\hline C10627 Ам & 69 & 0.51 & 0.49 \\
\hline C10627AN & 50 & 0.70 & 0.30 \\
\hline C10627AO & 35 & 0.00 & 1.00 \\
\hline C10627AP & 68 & 0.62 & 0.38 \\
\hline C10627AQ & 67 & 0.52 & 0.48 \\
\hline C10627AR & 39 & 0.67 & 0.33 \\
\hline C10627AS & 65 & 0.69 & 0.31 \\
\hline C10627AT & 83 & 0.34 & 0.66 \\
\hline C10627AU & 38 & 0.68 & 0.32 \\
\hline C10627AV & 73 & 0.36 & 0.64 \\
\hline C10627AW & 54 & 0.56 & 0.44 \\
\hline C10627AX & 71 & 0.54 & 0.47 \\
\hline C10627AY & 27 & 0.44 & 0.56 \\
\hline C10627AZ & 51 & 0.94 & 0.06 \\
\hline C10627BA & 74 & 0.53 & 0.47 \\
\hline C10627BB & 66 & 0.56 & 0.44 \\
\hline
\end{tabular}

Supplemental Table 1. Continued.

\begin{tabular}{|c|c|c|c|}
\hline \multirow[b]{2}{*}{$\underline{F_{2} \text { plant }}$} & \multirow[b]{2}{*}{ Total progenies (no.) } & \multicolumn{2}{|c|}{ Proportion of progenies } \\
\hline & & WT & MSC \\
\hline$\overline{\mathrm{C} 10627 \mathrm{BC}}$ & 47 & 0.70 & 0.30 \\
\hline C10627BD & 51 & 0.41 & 0.59 \\
\hline C10627BE & 28 & 0.64 & 0.36 \\
\hline $\mathrm{C} 10627 \mathrm{BF}$ & 70 & 0.00 & 1.00 \\
\hline C10627BG & 94 & 0.53 & 0.47 \\
\hline C10627BH & 38 & 0.61 & 0.40 \\
\hline C10627BI & 32 & 0.84 & 0.16 \\
\hline C10627BJ & 81 & 0.52 & 0.48 \\
\hline C10627BK & 54 & 0.02 & 0.98 \\
\hline C10627BL & 55 & 0.95 & 0.06 \\
\hline C10627BM & 27 & 0.67 & 0.33 \\
\hline $\mathrm{C} 10627 \mathrm{BN}$ & 48 & 0.46 & 0.54 \\
\hline C10627BO & 66 & 0.55 & 0.46 \\
\hline C10627BP & 73 & 0.52 & 0.48 \\
\hline C10627BQ & 72 & 0.07 & 0.93 \\
\hline C10627BR & 66 & 0.49 & 0.52 \\
\hline C10627BS & 54 & 0.04 & 0.96 \\
\hline C10627BT & 68 & 0.78 & 0.22 \\
\hline C10627BU & 27 & 0.56 & 0.44 \\
\hline C10627BV & 72 & 0.53 & 0.47 \\
\hline C10627BW & 59 & 0.56 & 0.44 \\
\hline C10627BX & 59 & 0.51 & 0.49 \\
\hline C10627BY & 62 & 0.47 & 0.53 \\
\hline C10627BZ & 78 & 0.64 & 0.36 \\
\hline C10627CA & 68 & 0.41 & 0.59 \\
\hline C10627CB & 74 & 0.01 & 0.99 \\
\hline C10627CC & 89 & 0.52 & 0.48 \\
\hline C10627CD & 72 & 0.49 & 0.51 \\
\hline C10627CE & 89 & 0.01 & 0.99 \\
\hline $\mathrm{C} 10627 \mathrm{CF}$ & 36 & 0.06 & 0.94 \\
\hline C10627CG & 76 & 0.55 & 0.45 \\
\hline $\mathrm{C} 10627 \mathrm{CH}$ & 78 & 0.00 & 1.00 \\
\hline C10627CI & 80 & 0.50 & 0.50 \\
\hline C10627CJ & 70 & 0.53 & 0.47 \\
\hline C10627CK & 38 & 0.53 & 0.47 \\
\hline C10627CL & 8 & 0.00 & 1.00 \\
\hline C10627CM & 82 & 0.04 & 0.96 \\
\hline $\mathrm{C} 10627 \mathrm{CN}$ & 46 & 0.76 & 0.24 \\
\hline $\mathrm{C} 10627 \mathrm{CO}$ & 16 & 0.06 & 0.94 \\
\hline C10627CP & 87 & 0.39 & 0.61 \\
\hline C10627CQ & 81 & 0.56 & 0.44 \\
\hline C10627CR & 17 & 0.00 & 1.00 \\
\hline C10627CS & 49 & 0.45 & 0.55 \\
\hline C10627CT & 90 & 0.01 & 0.99 \\
\hline C10627CU & 45 & 0.58 & 0.42 \\
\hline C10627CV & 60 & 0.08 & 0.92 \\
\hline C10627CW & 51 & 0.51 & 0.49 \\
\hline C10627CX & 71 & 0.38 & 0.62 \\
\hline C10627CY & 23 & 0.74 & 0.26 \\
\hline C10627CZ & 41 & 0.63 & 0.37 \\
\hline C10627DA & 80 & 0.40 & 0.60 \\
\hline C10627DB & 45 & 0.49 & 0.51 \\
\hline C10627DC & 77 & 0.01 & 0.99 \\
\hline C10627DD & 63 & 0.43 & 0.57 \\
\hline C10627DE & 90 & 0.61 & 0.39 \\
\hline
\end{tabular}

(Continued on next page) 
Supplemental Table 1. Continued.

\begin{tabular}{lccc}
\hline & & \multicolumn{2}{c}{ Proportion of progenies } \\
\cline { 3 - 4 } $\mathrm{F}_{2}$ plant & Total progenies (no.) & WT & MSC \\
\hline C10627DF & 96 & 0.48 & 0.52 \\
C10627DG & 81 & 0.57 & 0.43 \\
C10627DH & 58 & 0.53 & 0.47 \\
C10627DI & 86 & 0.65 & 0.35 \\
C10627DJ & 60 & 0.40 & 0.60 \\
C10627DK & 76 & 0.75 & 0.25 \\
C10627DL & 34 & 0.00 & 1.00 \\
C10627DM & 44 & 0.30 & 0.71 \\
C10627DN & 18 & 0.67 & 0.33 \\
C10627DO & 51 & 0.55 & 0.45 \\
C10627DP & 75 & 0.53 & 0.47 \\
C10627DQ & 42 & 0.60 & 0.41 \\
C10627DR & 69 & 0.58 & 0.42 \\
\hline
\end{tabular}

